\title{
Intrinsic thalamocortical connectivity varies in the age of onset subtypes in major depressive disorder
}

This article was published in the following Dove Medical Press journal:

Neuropsychiatric Disease and Treatment

\author{
Elliot C Brown ${ }^{1-3}$ \\ Darren L Clark ${ }^{1-3}$ \\ Stefanie Hassel ${ }^{1,2}$ \\ Glenda MacQueen ${ }^{1,2}$ \\ Rajamannar Ramasubbu ${ }^{1-3}$ \\ 'Mathison Centre for Mental \\ Health Research and Education, \\ University of Calgary, Calgary, AB, \\ Canada; ${ }^{2}$ Department of Psychiatry, \\ University of Calgary, Calgary, AB, \\ Canada; ${ }^{3}$ Department of Clinical \\ Neurosciences, University of Calgary, \\ Calgary, AB, Canada
}

Background: Differences in the thalamocortical system have been shown in patients with major depressive disorder (MDD). Given prior evidence of phenotypic heterogeneity by the age of onset in MDD, we examined whether differences in thalamocortical connectivity could identify biological subtypes of MDD defined by the age of illness onset.

Methods: A total of 94 subjects including 20 early-onset (EO) MDD (onset, 18 years), 34 adult-onset (AO) MDD, and 40 healthy controls (HCs) underwent resting-state functional MRI. Blood-oxygen-level-dependent time courses were extracted from six cortical regions of interest (ROIs) consisting of frontal, temporal, parietal, and occipital lobes and precentral and postcentral gyri. Each ROI's time course was then correlated with each voxel in thalamus, while covarying out signal from every other ROI.

Results: The analysis of variance results showed significant main effects of group in frontal and temporal connectivity with thalamus. Group contrasts showed a right fronto-thalamic hypoconnectivity only in AO-MDD, but not in EO-MDD, when compared to HCs. However, direct comparison between EO-MDD and AO-MDD showed no differences. Furthermore, there was a right temporal-thalamic hyperconnectivity in both EO-MDD and AO-MDD patients relative to HCs. These results were not accounted for by sex, age, or illness burden.

Conclusion: The age of illness onset may be a source of heterogeneity in fronto-thalamic intrinsic connectivity in MDD.

Keywords: depression, age of onset, neuroimaging, thalamus, cortex, resting-state fMRI

\section{Introduction}

Major depressive disorder (MDD) is a prevalent and disabling mental disorder characterized by a variety of emotional and cognitive impairments that are associated with changes in brain function. MDD is a highly heterogeneous condition, with much variability in symptom type, illness severity, and course. ${ }^{1}$ One source of heterogeneity that contributes to long-term morbidity is the age of onset of MDD. Preadult onset or early-onset (EO) MDD (under the age of 18 years) was found to be associated with greater illness severity, more depressive episodes, and increased suicidality as compared to patients with adult-onset (AO) MDD (onset age $\geq 18$ years), ${ }^{2}$ suggesting that the illness onset age could be representative of different clinical subtypes. Besides clinical features, findings from MRI studies have also shown to be distinct in EO-MDD compared with AO-MDD patients, further validating the clinical subtypes based on the onset age. Volumetric differences have been predominantly noted in genu of corpus callosum, subgenual cingulate cortex, and hippocampus and in cortical thickness of superior temporal cortex between EO-MDD and AO-MDD patients. ${ }^{3-5}$ Furthermore, fronto-striatal functional compensation during cognitive control was found to be less substantial in youth MDD patients compared with older individuals with MDD. ${ }^{6}$
Correspondence: Rajamannar Ramasubbu Mathison Centre for Mental Health Research \& Education, University of Calgary, TRW Building, Room 4D64, 3280 Hospital Drive NW, Calgary, AB T2N4Z6, Canada Email rramasub@ucalgary.ca
Neuropsychiatric Disease and Treatment 2019:15 75-82

(c) (1) (8) 2019 Brown et al. This work is published and licensed by Dove Medical Press Limited. The full terms of this license are avalable at https//www.dovepress.com/terms.php cc. hereby accept the Terms. Non-commercial uses of the work are permitted without any further permission from Dove Medical Press Limited, provided the work is properly attributed. For permission for commercial use of this work, please see paragraphs 4.2 and 5 of our Terms (https://www.dovepress.com/terms.php). 
The thalamus is a critical brain region implicated in depression and a hub for multiple streams of sensory, perceptual, cognitive, and emotional processing. ${ }^{7}$ The thalamocortical circuit has consistently been identified as the primary network associated with the underlying psychopathology of MDD. ${ }^{8-11}$ A number of studies have demonstrated substantial volumetric abnormalities in the cortico-striatalpallidal-thalamic circuits, fronto-thalamic white matter (WM) connectivity, and resting-state functional connectivity of thalamocortical circuits in MDD. ${ }^{12-16}$ One lesion study has shown an association between thalamic damage and cognitive and emotional deficits, highlighting a potentially causal link between disturbed thalamic function and MDD psychopathology. ${ }^{17}$

Previous work from our group has demonstrated abnormalities in thalamocortical connectivity in adult MDD patients. ${ }^{18}$ Our earlier study showed hyperconnectivity between medial thalamus and temporal regions and between medial thalamus and somatosensory areas in MDD patients compared with healthy controls (HCs). Furthermore, there was also a positive correlation between thalamotemporal connectivity and the severity of symptoms. This study extended our previous work in examining whether there is heterogeneity in thalamocortical connectivity based on the age of onset subtypes given that the neurodevelopment of thalamus and that of cortex are closely linked and that we know that alterations in the developmental trajectories of limbic and striatal regions are associated with depression onset. ${ }^{19,20}$ Ultimately, this work could later be used to identify thalamocortical circuit abnormalities as imaging markers of clinical subtypes and therefore could contribute to a better understanding of the underlying neurological changes in these subtypes to work toward more effective treatment targets.

The aim of this study was to use resting-state functional MRI (fMRI) to compare thalamocortical connectivity between EO-MDD ( $<18$ years) and AO-MDD ( $\geq 18$ years) in a group of medication-free MDD adult patients. We hypothesized that there would be regionally specific differences in thalamocortical connectivity between EO-MDD and AO-MDD patients, which would also differ from a group of HCs. More specifically, in the light of the findings from a systematic review of fMRI studies that cognitive abnormalities were more pronounced in late onset or AO than in EO-MDD,${ }^{21}$ we predicted that the most prominent group differences would be seen in functional connectivity between thalamus, and frontal and temporal regions as these circuits have been implicated in cognitive control in MDD. ${ }^{6}$

\section{Method}

\section{Participants}

In total, 54 patients diagnosed with MDD, according to the Structured Clinical Interview for the Diagnostic and Statistical Manual of Mental Disorders Axis-1 Disorders (SCID for DSM-IV), ${ }^{22}$ were recruited. The Hamilton Rating Scale for Depression (HAM-D) $)^{23}$ was administered to all the patients in order to determine the severity of depressive symptoms. The primary inclusion criteria were that the patients had to have received a score of $\geq 18$ on the HAM-D and had an acute episode of MDD. The patients were also required to be free from psychotropic medication for at least 3-4 weeks prior to recruitment. Three patients were medication-naïve, and all others had previously been exposed to antidepressant medication. Exclusion criteria included the presence of other Axis I disorders, history of substance abuse within the last 6 months, any neurological or unstable medical disorders, severe suicidal thoughts, or treatment resistance to three trials of different antidepressant medications. The patients were divided into two subgroups according to their age of illness onset, with an EO group (onset before the age of 18 years) and AO group (onset at or after the age of 18 years), with 20 in the EO group and 34 in the AO group. The study psychiatrist (RR) determined the age of onset based on participants' self-report on at what age they began to experience the constellation of symptoms that would fulfill the criteria for MDD.

A group of $40 \mathrm{HC}$ participants were also recruited and screened for Axis I psychiatric disorders, current or past, using the SCID for DSM-IV. Exclusion criteria included a family history of Axis-I disorders, a neurological disorder, or substance abuse within the last 6 months. The study was approved by the Conjoint Health Research Ethics Board of the University of Calgary. All the participants gave written informed consent before taking part, and all research procedures in this study were performed in accordance with the Declaration of Helsinki.

\section{MRI data acquisition}

MRI data were acquired across two different scanners. Resting-state fMRI blood-oxygen-level-dependent (BOLD) images from $45 \mathrm{MDD}$ and $18 \mathrm{HC}$ participants were collected using a 3T General Electric MR scanner (Signa VHi; General Electric Healthcare, Waukesha, WI, USA) using an eightchannel, phased-array head coil. Five minutes of resting data were acquired using a single-shot gradient-recalled echo, echo planar imaging (EPI) sequence (150 volumes, repetition time $[\mathrm{TR}] /$ echo time $[\mathrm{TE}]=2,000 / 30 \mathrm{~ms}, 24 \times 24 \mathrm{~cm}$ field of view, flip angle $=65^{\circ}, 30$ slices of $4 \mathrm{~mm}$ thickness). In addition, a T1-weighted structural MRI (TR $=9.2 \mathrm{~ms}, \mathrm{TE}=$ minimum, flip angle $=20^{\circ}, 180$ slices at a resolution of $1 \mathrm{~mm}$ ) was acquired 
for anatomical registration of the functional data. Resting-state fMRI BOLD images of nine MDD and $22 \mathrm{HC}$ participants were collected using 3T General Electric MR scanner (Discovery $^{\mathrm{TM}}$ MR750; General Electric Healthcare, Waukesha, WI, USA). Of these participants, the MDD patients had the same scanning parameters and protocol as that from the other scanner. All HC participants scanned on the Discovery scanner had a total of 5 minutes of resting-state data, acquired with an EPI sequence ( 119 volumes, TR/TE $=2,500 / 30 \mathrm{~ms}, 24 \times 24$ $\mathrm{cm}$ field of view, flip angle $=77^{\circ}, 40$ slices of $3 \mathrm{~mm}$ thickness). A T1-weighted structural MRI $(\mathrm{TR}=8.2 \mathrm{~ms}, \mathrm{TE}=$ minimum, flip angle $=18^{\circ}$, at a resolution of $1.3 \mathrm{~mm}$ ) was also acquired for anatomical registration. In all scans, the participants were asked to remain as still as possible in the MRI scanner with their eyes open and focused on a fixation cross at the center of the projection screen. They were asked to relax, not to think about anything in particular, and to stay awake.

\section{Image preprocessing and thalamocortical connectivity analysis}

Preprocessing of functional images included brain extraction, spatial smoothing with a Gaussian filter full-widthhalf-maximum of $5 \mathrm{~mm}$, and slice timing correction with a Hanning Window Sinc Interpolation and a temporal highpass filter of 100 seconds. Then, functional images were coregistered to individual subject's anatomical images and then to a standard Montreal Neurological Institute (MNI) template. Each participant's functional image was segmented into gray matter, WM, and cerebrospinal fluid (CSF) using manual tracing, of which the time series were later used as temporal covariates in the general linear model (GLM). The FMRIB Software Library ${ }^{24}$ was used for processing all the images. Global brain signal was not regressed out due to reports of the thalamus highly correlating with this signal. ${ }^{25}$

After preprocessing, six cortical regions of interest (ROIs) were defined using the Oxford Thalamic Connectivity Atlas, ${ }^{26}$ following a similar approach used by Zhang et al. ${ }^{25,27}$ This consisted of six cortical regions shown to have specific anatomical connections with thalamic regions, ie, 1) frontal, 2) motor (including primary and premotor), 3) somatosensory, 4) temporal, 5) parietal, and 6) occipital cortices. Figure $1 \mathrm{~A}$ illustrates these six ROIs. BOLD time courses were extracted from each cortical ROI and used as separate seeds in a seedto-voxel functional connectivity analysis to reveal correlations in activity between each cortical seed and each voxel in the thalamus. GLMs were created for each cortical seed where activity in every other cortical ROI was covaried out to identify the unique contribution in the functional connectivity of that particular cortical ROI. Movement parameters, WM, and
CSF time courses were also included in the GLMs as variables of no interest in the first-level analysis. As we used two different scanners to acquire the MRI data, we included scanner and age and sex in the GLMs as variables of no interest in the second-level analysis to control for their potential confounding effects. Group mean statistical maps of functional connectivity were then created for each cortical ROI, for each group (HC, EO-MDD, and AO-MDD), showing connectivity between each cortical ROI and each voxel in the thalamus. To have a more conservative and thus more robust test for group differences, an $F$-test (analysis of variance [ANOVA]) was performed with all three groups' statistical maps to identify clusters that showed main group effects. Following this, post hoc analyses were performed in which two group contrasts were performed to reveal the direction of effects and the group that drove the effects. Only the clusters that emerged from the group contrasts that overlapped with clusters identified in the ANOVA result were considered to be significant.

Figure 1 shows all statistical maps (group means, ANOVA main effects, and group contrasts), which were thresholded at a voxel-wise $\alpha$ level of $P<0.005$. Correction for multiple comparisons was done for group means and contrasts using small volume correction with the SPM WFU Pickatlas toolbox ${ }^{28}$ using a corrected $\alpha$ level of $P=0.001$ family-wise error (cluster size $>6$ voxels), considering only voxels within the Harvard-Oxford thalamus atlas. ${ }^{26}$

\section{Results}

Table 1 shows the demographic and clinical characteristics of patients in each group. The age, sex, age of MDD onset, and the number of episodes significantly differed between groups.

\section{Thalamocortical connectivity in EO-MDD/ AO-MDD groups}

Figure 1B displays statistical maps for group means for $\mathrm{HC}$, EO-MDD, and AO-MDD groups and main effects from the ANOVA results comparing the three groups. Significant main effects of groups emerged from the ANOVA between frontal (MNI coordinates for local maxima: $\mathrm{x}=10, \mathrm{y}=-22$, $\mathrm{z}=4$; cluster size $=4 ; z$-score $=2.69)$ and temporal $(\mathrm{MNI}$ coordinates for local maxima: $\mathrm{x}=18, \mathrm{y}=-32, \mathrm{z}=-8$; cluster size $=12 ; z$-score $=3.03$ ) connectivity with thalamus. More specifically, the main effect of the group in thalamofrontal connectivity was localized in the right mediodorsal/midline thalamic nucleus, and the main effect of group in thalamotemporal connectivity was localized in the right posterior ventral part of the pulvinar. The fourth row of Figure 1B displays the results from the ANOVA and the regions of connectivity within the thalamus showing the main effects of the group. 
A
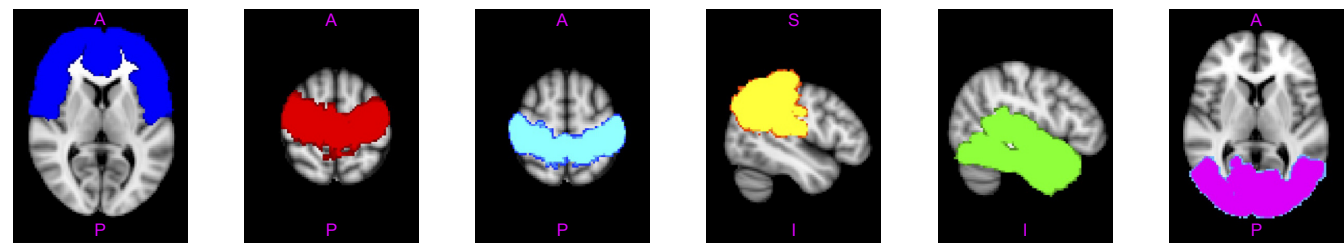

Frontal
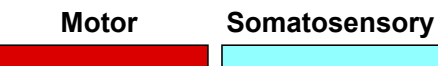

Perietal

Temporal

Occipital

B
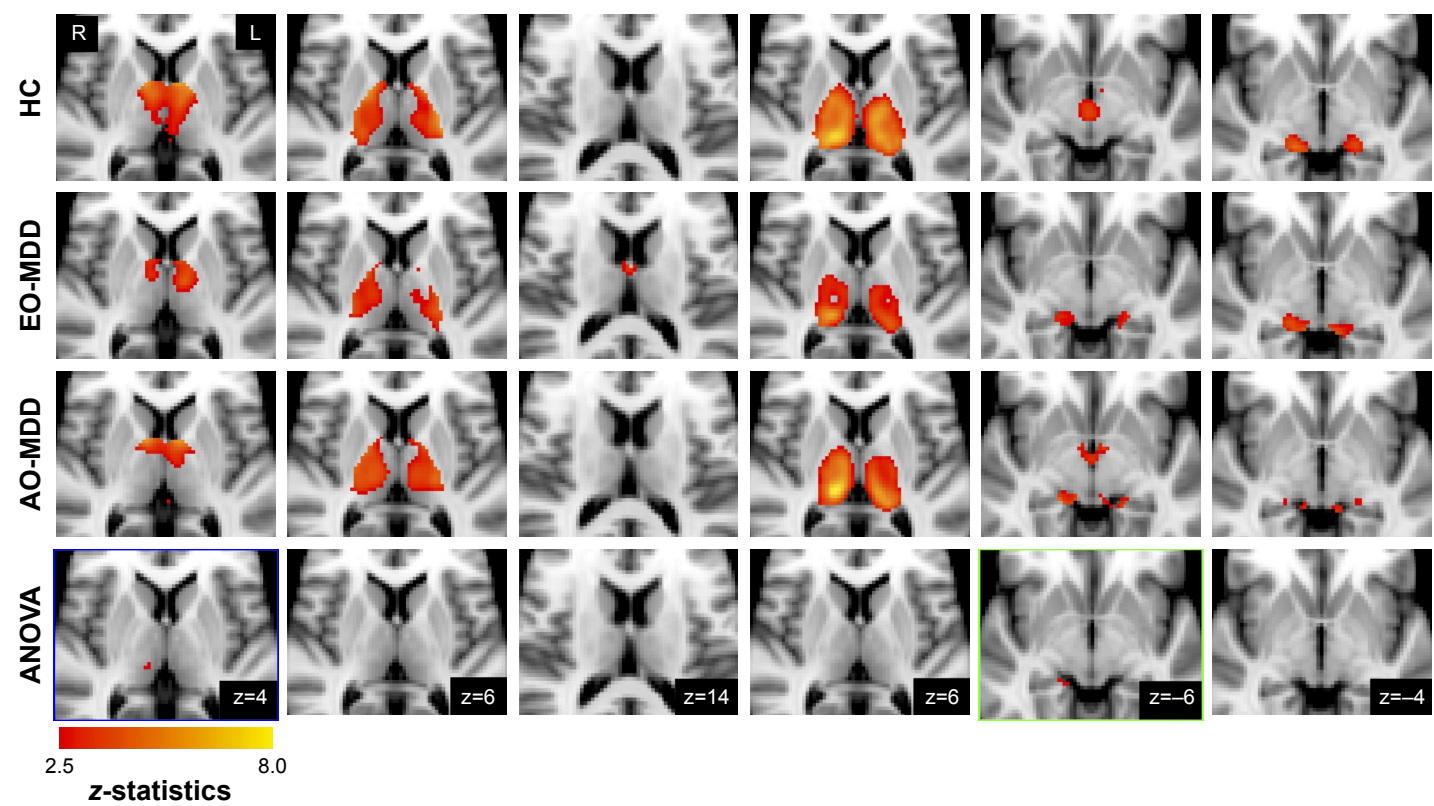

C

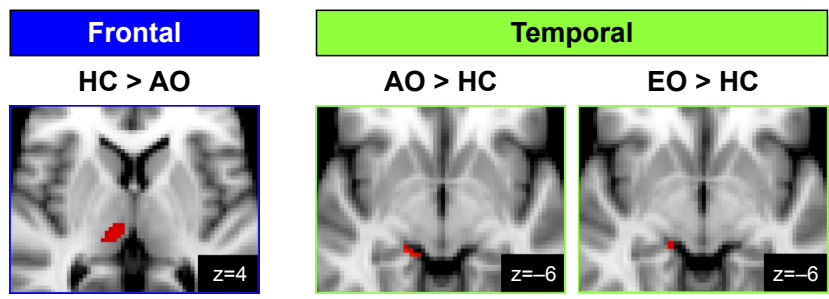

D
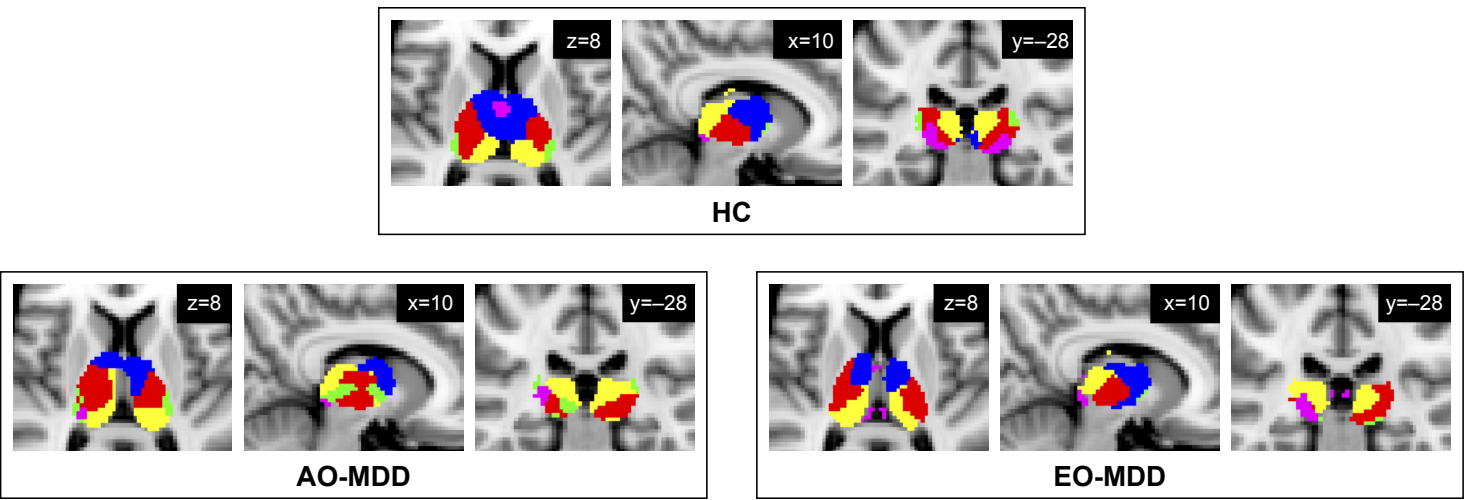

Figure I Statistical maps of thalamocortical connectivity in EO-MDD and AO-MDD patients and HC: (A) six cortical ROls used as seed regions in the seed-to-voxel analysis of thalamocortical connectivity; (B) the first three rows show group mean clusters from HCs and EO-MDD and AO-MDD patients. The fourth row displays significant clusters showing a main effect of group from ANOVA results; (C) significant clusters emerging from group contrasts (showing only clusters that overlap with group main effects from ANOVA results); (D) overlaid group mean clusters to demonstrate differences in the spatial distribution of thalamocortical connectivity regions in the thalamus across all the three groups.

Note: All statistical maps corrected at a voxel level of $P<0.005$ and after small volume correction (FWE), $P<0.00$ I.

Abbreviations: ANOVA, analysis of variance; AO, adult onset; $\mathrm{EO}$, early onset; $\mathrm{FWE}$, family-wise error; $\mathrm{HC}$, healthy control; $\mathrm{MDD}$, major depressive disorder; ROIs, regions of interest. 
Table I Demographic and clinical variables

\begin{tabular}{|l|l|l|l|l|l|}
\hline & $\begin{array}{l}\text { EO-MDD } \\
(\mathbf{N}=\mathbf{2 0})\end{array}$ & $\begin{array}{l}\text { AO-MDD } \\
\mathbf{( N = 3 4 )}\end{array}$ & $\begin{array}{l}\text { Healthy controls } \\
\mathbf{( N = 4 0 )}\end{array}$ & $\begin{array}{l}\text { F-statistics } \\
\text { (EO vs AO) }\end{array}$ & P-values \\
\hline Age (years) & $29.2 \pm 7.9$ & $41.1 \pm 9.4$ & $31.3 \pm 9.7$ & $22.68^{* * *}$ & $<0.001$ \\
\hline Sex (\%) & $25 \mathrm{M}, 75 \mathrm{~F}$ & $44 \mathrm{M}, 56 \mathrm{~F}$ & $45 \mathrm{M}, 55 \mathrm{~F}$ & $5.04^{*}$ & 0.029 \\
\hline HAM-D total & $21.8 \pm 3.5$ & $21.6 \pm 4.3$ & - & 0.05 & $0.83 \mathrm{I}$ \\
\hline Age of onset & $14.4 \pm 2.5$ & $31.6 \pm 8.1$ & - & $85.17^{* * *}$ & $<0.001$ \\
\hline Number of episodes & $4.6 \pm 5.8$ & $1.7 \pm 1.6$ & - & $7.46^{* *}$ & 0.009 \\
\hline Illness duration (months) & $59.5 \pm 74.2$ & $41.9 \pm 55.0$ & - & 0.99 & 0.324 \\
\hline
\end{tabular}

Note: $* P<0.05 ; * * P<0.01$; *** $P<0.001$.

Abbreviations: AO, adult onset; EO, early onset; F, female; M, male; MDD, major depressive disorder; HAM-D, Hamilton Rating Scale for Depression.

\section{Age of onset and thalamocortical connectivity}

The post hoc analysis of group contrasts in regions that showed significant effects from the $F$-test showed a significantly decreased connectivity between right mediodorsal/ midline thalamus and frontal cortices in the AO-MDD group, but not in the EO-MDD group, when compared to HCs. Second, both AO-MDD and EO-MDD groups exhibited an increased connectivity between right posterior ventral thalamus and temporal cortices. It is worth noting that the size of the cluster that showed a hyperconnectivity between temporal cortices and thalamus was larger in the AO-MDD group, when compared to the EO-MDD group. Table 2 lists the significant clusters, and Figure $1 \mathrm{C}$ shows the statistical maps for significant illness onset effects. Figure 1D shows group mean clusters overlaid on one another for HC, EO-MDD, and AO-MDD groups, in order to illustrate the spatial distribution of thalamocortical connectivity patterns.

In order to control for illness burden, and as we see a significant difference in the number of depressive episodes between EO-MDD and AO-MDD groups, we performed an additional analysis using the number of episodes as a variable of no interest. After controlling for the number of episodes, we did not see any substantial change in the main ANOVA results or group contrasts. We also checked for correlations between age and $z$-scores of thalamofrontal and thalamotemporal connectivity $(\mathrm{r}(53)=0.051, P=0.713 ; \mathrm{r}(53)=0.009$, $P=0.948$, respectively), in order to demonstrate minimal confounding effects of age on our results.

\section{Discussion}

In this study, we sought to investigate whether there are differences in the thalamocortical connectivity between EO-MDD and AO-MDD patients and how each group differed from HCs. Our main findings first showed reduced connectivity between frontal cortex and medial thalamus only in AO-MDD patients, but not in the EO-MDD group, when compared to HC. Second, both AO-MDD and EOMDD groups showed a greater thalamotemporal connectivity between temporal cortex and posterior thalamus when compared to HCs. Another notable finding was that the effects were not accounted for by the current age, sex, or illness burden, suggesting that the effects may have been specific to the age of onset per se. Our results have shown

Table 2 Cluster table showing thalamocortical group differences from group contrasts (only clusters that overlap with those showing the main effects of the group from ANOVA comparing HC, AO-MDD, and EO-MDD patients)

\begin{tabular}{|c|c|c|c|c|c|c|}
\hline \multirow{2}{*}{$\begin{array}{l}\text { Region } \\
\text { (clusters) }\end{array}$} & \multirow{2}{*}{$\begin{array}{l}\mathbf{R}=\text { right; } \\
\mathbf{L}=\text { left }\end{array}$} & \multirow{2}{*}{$\begin{array}{l}\text { Cluster size } \\
\text { (voxels) }\end{array}$} & \multicolumn{3}{|c|}{ MNI coordinate (local maxima) } & \multirow[t]{2}{*}{ z-score } \\
\hline & & & $x$ & $y$ & $\mathbf{z}$ & \\
\hline \multicolumn{7}{|l|}{ EO effects } \\
\hline \multicolumn{7}{|l|}{$\mathrm{EO}>\mathrm{HC}$} \\
\hline $\begin{array}{l}\text { Temporal } \\
\text { AO effects }\end{array}$ & $\mathrm{R}$ & 10 & 16 & -32 & -8 & 3.3 \\
\hline \multicolumn{7}{|l|}{$\mathrm{AO}>\mathrm{HC}$} \\
\hline Temporal & $\mathrm{R}$ & 36 & 10 & -34 & -2 & 3.31 \\
\hline \multicolumn{7}{|l|}{$\mathrm{HC}>\mathrm{AO}$} \\
\hline Frontal & $\mathrm{R}$ & 67 & 10 & -22 & 4 & 3.32 \\
\hline
\end{tabular}

Abbreviations: ANOVA, analysis of variance; AO, adult onset; EO, early onset; HC, healthy control; MDD, major depressive disorder; MNI, Montreal Neurological Institute. 
both differential and shared abnormalities in thalamocortical connectivity in EO-MDD and AO-MDD. The differences in thalamofrontal connectivity may help to validate clinical subtypes based on the illness onset age.

Taking into account the direct relationship between dorsomedial thalamus-prefrontal abnormalities and cognitive impairment, ${ }^{29}$ the observed decreased connectivity between frontal cortex and thalamus in AO-MDD is partly in agreement with the reported greater cognitive impairment in AO-MDD relative to youth MDD. ${ }^{21}$ This thalamofrontal hypoconnectivity seems to be driven by the AO group as this finding was much weaker and emerged only at a lower threshold than in our previous study that investigated MDD adult patients as a whole group including both EO-MDD and AO-MDD patients. ${ }^{18}$ It is noteworthy that this finding could not be accounted for higher age in AO-MDD than in EO-MDD or HC, and therefore, it is more likely related to the adult age of onset in MDD. However, it remains unclear why this abnormality was absent in EO-MDD. One possibility is that prolonged exposure to antidepressants in the context of longer duration of illness in EO-MDD than in AO-MDD might have normalized this abnormality in EOMDD. Another possibility is that thalamic abnormalities may be rare in EO-MDD as the neurodevelopmental trajectory suggests nonlinear increases in thalamic volumes during childhood and adolescence and linear volumetric decreases in thalamus starting in the early adulthood continuing into the old age. ${ }^{30}$ This might explain why the volumetric reductions were more pronounced in late-onset MDD than in EO-MDD. ${ }^{12}$ In addition, the structural and neurochemical abnormalities in thalamus were reported in pediatric patients with obsessive-compulsive disorder but not in pediatric patients with MDD. ${ }^{31,32}$

The increased connectivity between thalamus and temporal cortex in both EO-MDD and AO-MDD groups may reflect impaired emotional memory adaptation and memory modulations through thalamo-amygdala ${ }^{33}$ and thalamohippocampal pathways ${ }^{34}$ and impaired social cognition through thalamic connections with anterior temporal lobes. ${ }^{35}$ This shared abnormality in thalamotemporal hyperconnectivity may also subserve the severity of illness ${ }^{18}$ and negative bias in MDD, ${ }^{36,37}$ which occur as a universal characteristic of depressive psychopathology, regardless of the age of onset.

\section{Limitations}

The main limitation of the study was the use of retrospective self-reports of age of onset of MDD, which we cannot guarantee were absolutely accurate. The recall bias might have caused overdiagnosis or false diagnosis of MDD, especially in the EO group. Hence, it is possible that the patients who had a less severe form of depression (subclinical depression) and high neuroticism might have been included in the EOMDD group. As discussed in the previous section, we cannot rule out the possible effects of long-term antidepressant use on thalamocortical connectivity. However, all the participants had refrained from taking medication in 3-5 weeks leading up to the scanning; therefore, we can at least say that the results were not driven by acute drug effects. Furthermore, our results showed some overlap between cortical seed regions used in the ROI analysis, which may have accounted for the lack of significant clusters in connectivity between somatosensory cortex and thalamus. Future studies may seek to identify smaller subregions of thalamocortical connectivity to look for more localized abnormalities in MDD. There was a substantial difference in age between groups in our study, and although we tried to control for this by including age as a covariate of no interest, we still may have not fully accounted for all the potential confounding effects of age. We did use two scanners to collect the imaging data, but believe that the effects of scanner changes were minimal, especially as we used a similar scanning protocol across scanners, and we used scanner as a covariate of no interest in our analysis. Given these methodological limitations and small sample size, our findings should be considered preliminary until replicated in a large sample. Our research question would also benefit from future longitudinal studies that could have the potential for finding more causal links between changes in thalamocortical connectivity and the age of illness onset in depression. However, here, we presented a first step that could provide crucial clues for further investigation.

\section{Conclusion}

We first demonstrated that AO-MDD exhibited a specific thalamofrontal hypoconnectivity not seen in EO-MDD, which may be a marker for dissociating EO-MDD and AO-MDD subtypes. Second, we showed thalamotemporal hyperconnectivity in both EO-MDD and AO-MDD groups, which appears to be not related to the age of onset, but more generally related to the underlying psychopathology of MDD. Our study provides a further support to investigating the neural underpinnings of MDD subgroups based on the age of illness onset.

\section{Acknowledgments}

We acknowledge the contribution of Bradley Goodyear, Ismael Gaxiola, and Filomeno Cortese from the Seaman Family MR Research Center for imaging data acquisition, storage, and quality assurance. The authors wish to thank all 
the participants who volunteered their time for this study. This work was supported by an investigator initiated grant from Astra Zeneca awarded to Dr Ramasubbu. The funding agency (Astra Zeneca) had no role in the design of the study, collection, and analysis of data and in the decision to publish it. He also received an investigator initiated grant from Pfizer. He has served as a member of the advisory committee for Lundbeck and Janssen and received honorarium. Dr MacQueen has received honoraria from Pfizer, Janssen, Lilly, Allergen, and Lundbeck. The authors Dr Brown and Dr Clark are currently supported by fellowships from Alberta Innovates Health Solutions, and Dr Brown was supported by a fellowship from the Mathison Centre for Mental Health Research \& Education for a part of the duration of data analysis and manuscript preparation.

\section{Disclosure}

This work was supported by investigator initiated grant by Astra Zeneca to Dr. Rajamannar Ramasubbu. The authors report no other conflicts of interest in this work.

\section{References}

1. Merikangas KR, Wicki W, Angst J. Heterogeneity of depression. Classification of depressive subtypes by longitudinal course. Br J Psychiatry J Ment Sci. 1994;164:342-348.

2. Zisook S, Rush AJ, Albala A, et al. Factors that differentiate early vs. later onset of major depression disorder. Psychiatry Res. 2004;129(2): 127-140.

3. Kemp A, Macmaster FP, Jaworska N, et al. Age of onset and corpus callosal morphology in major depression. $J$ Affect Disord. 2013;150(2): 703-706.

4. Jaworska N, Macmaster FP, Yang X-R, et al. Influence of age of onset on limbic and paralimbic structures in depression. Psychiatry Clin Neuroscis. 2014;68(12):812-820.

5. Jaworska N. A preliminary study of the influence of age of onset and childhood trauma on cortical thickness in major depressive disorder. BioMed Res Int. 2014.

6. Rao JA, Kassel MT, Weldon AL, et al. The double burden of age and major depressive disorder on the cognitive control network. Psychol Aging. 2015;30(2):475-485.

7. Haber SN. The primate basal ganglia: parallel and integrative networks. J Chem Neuroanat. 2003;26(4):317-330.

8. Drevets WC, Videen TO, Price JL, Preskorn SH, Carmichael ST, Raichle ME. A functional anatomical study of unipolar depression. J Neurosci. 1992;12(9):3628-3641.

9. Price JL, Drevets WC. Neurocircuitry of mood disorders. Neuropsychopharmacology. 2010;35(1):192-216.

10. Shepherd GM. Corticostriatal connectivity and its role in disease. Nat Rev Neurosci. 2013;14(4):278-291.

11. Swerdlow NR, Koob GF. Dopamine, schizophrenia, mania, and depression: toward a unified hypothesis of cortico-striato-pallido-thalamic function. Behavioral and Brain Sciences. 1987;10(02):197-208.

12. Bora E, Harrison BJ, Davey CG, Yücel M, Pantelis C. Meta-analysis of volumetric abnormalities in cortico-striatal-pallidal-thalamic circuits in major depressive disorder. Psychol Med. 2012;42(4):671-681.

13. Webb CA, Weber M, Mundy EA, Killgore WD. Reduced gray matter volume in the anterior cingulate, orbitofrontal cortex and thalamus as a function of mild depressive symptoms: a voxel-based morphometric analysis. Psychol Med. 2014;44(13):2833-2843.
14. Greicius MD, Flores BH, Menon V, et al. Resting-state functional connectivity in major depression: abnormally increased contributions from subgenual cingulate cortex and thalamus. Biol Psychiatry. 2007;62(5): 429-437.

15. Jia Z, Wang Y, Huang $X$, et al. Impaired frontothalamic circuitry in suicidal patients with depression revealed by diffusion tensor imaging at 3.0 T. J Psychiatry Neurosci. 2014;39(3):170-177.

16. Lui $\mathrm{S}, \mathrm{Wu} \mathrm{Q}$, Qiu L, et al. Resting-state functional connectivity in treatment-resistant depression. Am J Psychiatry. 2011;168(6):642-648.

17. Liebermann D, Ostendorf F, Kopp UA, et al. Subjective cognitiveaffective status following thalamic stroke. $J$ Neurol. 2013;260(2): 386-396.

18. Brown EC, Clark DL, Hassel S, Macqueen G, Ramasubbu R. Thalamocortical connectivity in major depressive disorder. $J$ Affect Disord. 2017;217:125-131.

19. Whittle S, Lichter R, Dennison M, et al. Structural brain development and depression onset during adolescence: a prospective longitudinal study. Am J Psychiatry. 2014;171(5):564-571.

20. Jones EG. The Thalamus. Cambridge: Cambridge University Press; 2007.

21. Kerestes R, Davey CG, Stephanou K, Whittle S, Harrison BJ. Functional brain imaging studies of youth depression: a systematic review. Neuroimage Clin. 2014;4:209-231.

22. First M, Gibbon M, Williams J. Structured Clinical Interview for DSM-IV-TR Axis I Disorders, Research Version, Patient Edition. (SCID$I / P)$. New York, NY: Biometric Research, New York State Psychiatric Institute; 2002.

23. Hamilton M. Development of a rating scale for primary depressive illness. Br J Soc Clin Psychol. 1967;6(4):278-296.

24. Smith SM, Jenkinson M, Woolrich MW, et al. Advances in functional and structural MR image analysis and implementation as FSL. Neuroimage. 2004;23(Suppl 1):S208-S219.

25. Zhang D, Snyder AZ, Fox MD, Sansbury MW, Shimony JS, Raichle ME. Intrinsic functional relations between human cerebral cortex and thalamus. J Neurophysiol. 2008;100(4):1740-1748.

26. Behrens TE, Johansen-Berg H, Woolrich MW, et al. Non-invasive mapping of connections between human thalamus and cortex using diffusion imaging. Nat Neurosci. 2003;6(7):750-757.

27. Zhang D, Snyder AZ, Shimony JS, Fox MD, Raichle ME. Noninvasive functional and structural connectivity mapping of the human thalamocortical system. Cereb Cortex. 2010;20(5):1187-1194.

28. Maldjian JA, Laurienti PJ, Kraft RA, Burdette JH. An automated method for neuroanatomic and cytoarchitectonic atlas-based interrogation of fMRI data sets. Neuroimage. 2003;19(3):1233-1239.

29. Parnaudeau S, Bolkan SS, Kellendonk C. The mediodorsal thalamus: an essential partner of prefrontal cortex for cognition. Biol Psychiatry. 2017;83(8):648-656.

30. Walhovd KB, Fjell AM, Reinvang I, et al. Effects of age on volumes of cortex, white matter and subcortical structures. Neurobiol Aging. 2005;26(9):1261-1270.

31. Kim JJ. Grey matter abnormalities in obsessive-compulsive disorder: statistical parametric mapping of segmented magnetic resonance images. Br J Psychiatry J Ment Sci. 2001;179:330-334.

32. Smith EA, Russell A, Lorch E, et al. Increased medial thalamic choline found in pediatric patients with obsessive-compulsive disorder versus major depression or healthy control subjects: a magnetic resonance spectroscopy study. Biol Psychiatry. 2003;54(12):1399-1405.

33. Romanski LM, Ledoux JE. Equipotentiality of thalamo-amygdala and thalamo-cortico-amygdala circuits in auditory fear conditioning. J Neurosci. 1992;12(11):4501-4509.

34. Vertes RP, Hoover WB, Szigeti-Buck K, Leranth C. Nucleus reuniens of the midline thalamus: link between the medial prefrontal cortex and the hippocampus. Brain Res Bull. 2007;71(6):601-609.

35. Olson IR, Mccoy D, Klobusicky E, Ross LA. Social cognition and the anterior temporal lobes: a review and theoretical framework. Soc Cogn Affect Neurosci. 2013;8(2):123-133. 
36. Hao L, Yang J, Wang Y, et al. Neural correlates of causal attribution in negative events of depressed patients: evidence from an fMRI study. Clin Neurophysiol. 2015;126(7):1331-1337.
37. Seidel EM, Satterthwaite TD, Eickhoff SB, et al. Neural correlates of depressive realism - an fMRI study on causal attribution in depression. J Affect Disord. 2012;138(3):268-276.

\section{Publish your work in this journal}

Neuropsychiatric Disease and Treatment is an international, peerreviewed journal of clinical therapeutics and pharmacology focusing on concise rapid reporting of clinical or pre-clinical studies on a range of neuropsychiatric and neurological disorders. This journal is indexed on PubMed Central, the 'PsycINFO' database and CAS,

and is the official journal of The International Neuropsychiatric Association (INA). The manuscript management system is completely online and includes a very quick and fair peer-review system, which is all easy to use. Visit http://www.dovepress.com/testimonials.php to read real quotes from published authors.

Submit your manuscript here: http://www.dovepress.com/neuropsychiatric-disease-and-treatment-journal 